\title{
Getting ready for malaria elimination: a check list of critical issues to consider
}

\author{
Matiana González-Silva ${ }^{1,2}{ }^{+}$, Quique Bassatt ${ }^{1,2,3}$, Pedro L Alonso ${ }^{1,2,3}$ \\ 'Barcelona Institute for Global Health, Barcelona, Spain ${ }^{2}$ Clinics Hospital, Barcelona Centre for International Health Research, \\ University of Barcelona, Barcelona, Spain ${ }^{3}$ Centro de Investigação em Saúde da Manhiça, Maputo, Moçambique
}

\begin{abstract}
In recent years, a renewed interest in malaria elimination and eradication has emerged and seems to be rooting in the minds of the scientific community, public health specialists, funding bodies, policy makers and politicians. Malaria eradication will certainly benefit from improved and innovative tools; notwithstanding novel knowledge in fields ranging from basic science to mathematical modelling and health systems research. However, the elimination of malaria also encompasses a broad range of essential aspects that countries and other actors need to consider when thinking of embarking on such an adventure, including the implementation of innovative strategies, the ability to incorporate the most up-to-date evidence into policy, the integration of malaria into the broader health agenda, the strengthening of surveillance and health systems, capacity building, funding, advocacy and, very importantly, research. While in some cases this enthusiasm is clearly justified, some countries are still a long way from realistically advancing towards elimination. This paper attempts to provide guidance on all the necessary issues that should be considered when initiating a malaria elimination program.
\end{abstract}

Key words: malaria - elimination - science - policy - health systems - training

In recent years, a renewed interest in malaria elimination and eradication has emerged and seems to be rooting in the minds of the scientific community, public health specialists, funding bodies, policy makers and politicians. Following the 2007 call for malaria eradication (Bill \& Melinda Gates Foundation 2007) and the publication of the Malaria Eradication Research Agenda (malERA 2011), an increasing number of scientific projects that specifically address the problems posed by the elimination of malaria have been put in place (Policy Cures 2013), accompanied by scientific coordination mechanisms, such as the Malaria Eradication Scientific Alliance (MESA 2011). We also have witnessed political declarations committing countries towards the elimination goal, together with novel regional elimination initiatives (Panel 1) in Central America and the Caribbean, South East Asia and southern Africa among others, while sections devoted to the elimination and eradication of malaria increasingly appear more often in technical guidelines and justifications for malaria grants.

These events are certainly a reason for celebration, as there seems to be general agreement that elimination is the only sustainable approach against malaria. Defined as the complete disappearance of plasmodia parasites that affect human beings in a given geographical area, elimination overcomes the threat of resurgences that too often follow a period in which malaria transmission has decreased to

doi: 10.1590/0074-0276140205

+ Corresponding author: matiana.gonzalez@isglobal.org

Received 7 June 2014

Accepted 18 July 2014 a point in which it ceases to be a public health concern and efforts become more relaxed (Newman 2012). In addition to making the maintenance of control interventions unnecessary (although requiring strong surveillance systems to avoid the reintroduction of infection), elimination may also be the solution to the menace foreseen by many modellers according to whom, even if we maintain current malaria intervention levels, the malaria burden still will rise and the loss of immunity in populations will progressively make them more vulnerable.

There are more than enough arguments to encourage all actors within the malaria community to embrace the goal of elimination quickly. However, beyond the evidence on the benefits of regional elimination and the ultimate eradication of malaria, it is also certain that such an ambitious goal will by no means come without challenges. Elimination requires strategies specifically aimed at the interruption of transmission and the clearance of parasites from asymptomatic carriers, a substantial modification to the traditional approach in malaria control. There is also a need to establish reliable surveillance systems to guide interventions as well as strong political commitment and sustained funding until the very end, most importantly during the so-called "last mile", when each infection is crucial to definitely interrupt transmission while, at the same time, being harder to find and proportionately much more expensive to tackle (Mendis et al. 2009).

The aim of this paper is not to pinpoint the previously stated knowledge gaps and the research and development needed to achieve malaria elimination and eradication, but rather to contribute to a better understanding of how we can prepare ourselves to move towards these goals. As indicated by the malERA papers, malaria eradication will certainly benefit from improved and innovative tools, notwithstanding novel knowledge in fields rang- 
ing from basic science to mathematical modelling and health systems research. However, the elimination of malaria also encompasses a broad range of essential aspects that countries and other actors need to take into consideration when thinking of embarking on such an adventure, including the implementation of innovative strategies and the ability to incorporate the most up-to-date evidence into policy, the integration of malaria into the broader health agenda, the strengthening of surveillance and health systems, capacity building, funding, advocacy and, very importantly, research (Breman et al. 2011).

In this paper, we openly reflect on the key elements we think are necessary to achieve malaria elimination wherever feasible and on advancing towards this longterm goal in areas in which the primary objective remains the control of the disease, albeit preparation for elimination may run in parallel (Moonen et al. 2010).

Tools and strategies for malaria elimination - Smallpox, the only human disease that has ever disappeared by the deliberate action of man, was eradicated thanks to the existence of a highly efficacious preventive tool (a vaccine) and the biological advantage of a close correlation between disease and infection. Unfortunately, in the case of malaria, neither of those features exists. The only available vaccine likely to be registered by 2015 , has an efficacy of $30-50 \%$ in African children (RTS,S Clinical Trials Partnership 2012) and other currently existing vector control strategies for malaria prevention leave open an enormous window for transmission to occur outdoors or when people are not sleeping. Additionally, in highly endemic countries, the acquisition of immunity against plasmodia naturally occurs throughout infancy and childhood and after this age new infections are often asymptomatic, representing large pools of apparently healthy malaria parasite carriers that can sustain transmission even when the number of deaths and clinical cases has dramatically fallen.

It is true that we lack the ideal tools to eliminate malaria (Alonso et al. 2011), but it is also true that with those we have available, we can go very far. The existing tools will need to be creatively used in combination with the newly developed ones. Experts will need to define the technical aspects of their implementation, including which drugs and diagnostics to use in combination with which preventive tools and whether those strategies need to reach populations at-risk as a whole or in a more targeted manner.

When we aim at completely clearing parasites from a given geographic area, the asymptomatic parasite prevalence may appear as an unsurmountable challenge and will therefore require aggressive strategies specifically aimed at finding those infections. Strategies to clear these "silent" parasites may include massive screening campaigns followed by radical cure treatment for infected individuals or through directly treating all members of the population at-risk with an effective antimalarial to avoid the need for screening. Better and more sensitive diagnostic tools, especially those identifying carriers of a low parasite burden or those not requiring blood sampling for malaria confirmation, may represent important advantages, not only for the early treatment of parasite carriers, but also for the prevention of reintroduction and the maintenance of zero transmission.
Linked to asymptomatic cases and equally important is addressing transmission as the key event to be prevented by an elimination campaign. In addition to new tools specifically designed with this goal in mind, such as vaccines that trigger immune responses against transmission stages of the malaria parasites, we need to efficiently utilise the existing tools, including drugs that kill stage $\mathrm{V}$ Plasmodium falciparum gametocytes (the ultimate stage responsible for transmission from humans to the mosquito vector), as well as improved vector control interventions.

The third pillar of malaria elimination relies on the surveillance system, as passive case detection embedded in health facilities may not be sufficient to achieve this goal. Strategies should therefore incorporate an active search for infected individuals and/or close case contacts that may also be tested and treated in the case of infection. Surveillance as a response then becomes a pivotal element of any campaign and should be considered as important as any other intervention or even an intervention per se.

All of these strategic elements should consider not only the elimination of P. falciparum, but also of Plasmodium vivax, the most common parasite outside Africa. $P$. vivax has some biological features that increase the difficulties for elimination and that surely require specific approaches. The crucial challenge for eliminating $P$. vivax are the asymptomatic carriers of liver stages of the parasite that may cause relapses months or even years following the original infection. Such hypnozoites can currently be treated only with primaquine, a drug that poses safety problems in individuals with glucose6-phosphate dehydrogenase deficiency, compromising its use in mass drug administration campaigns. In this context, vivax-endemic communities should be managed through aggressive campaigns to lower the malaria burden, combined with active surveillance to identify the very last clinical infections as they appear. Other species of malaria affecting humans represent a much lower public health problem, while the zoonotic species Plasmodium knowlesi, transmitted from macaques to men and therefore much more challenging to eliminate, is fully sensitive to chloroquine (CQ) and very circumscribed from a geographical point of view.

However, although elimination approaches in different settings may have some common elements, we need to keep in mind that no single approach is valid for all elimination scenarios. As clearly shown by the World Health Organization (WHO) Global Malaria Eradication Program of the mid-XX century, campaigns only will be successful if we go beyond a merely programmatic approach and we incorporate the creation of novel knowledge throughout the path towards the last infection (Nájera et al. 2011, RBM Partnership 2011).

This is the operational research component of elimination strategies, which could actually be best classified as an "investigation to solve problems that programs are actually facing". Operational research is too often perceived by countries as a way of diverting both human and financial resources to produce knowledge that only satisfies the intellectual curiosity of scientists. Nothing could be further from the truth; rather, this element denotes the capacity for understanding issues that for any reason are hindering the successful achievement of ex- 
pected outcomes and answering questions that will ultimately inform and guide actions in the field. To give one example, operational research could find out why an area proves to be "intervention resistant", even if all the supposedly correct actions are being implemented. Is malaria persistence due to insecticide resistance or is it a matter of mosquito behaviour? Although there are many other crucial questions to be asked (WHO 2014).

These programs should investigate not only the biological aspects of malaria transmission, but also the social and cultural determinants that influence the burden and spread of the disease, including human behaviour, language barriers, education, communication, acceptability and adherence to interventions and the particular idiosyncrasies of hard to reach populations, among others. This social sciences component is particularly relevant in remote indigenous areas, where malaria is often concentrated as countries approach elimination.

However, the generation of novel knowledge may still be insufficient for reaching malaria elimination. We need to make sure that the evidence generated by local and international research efforts is actually incorporated into the malaria programs. The WHO already has a Malaria Policy Advisory Committee, which revises the most up-to-date evidence and advises the WHO Director General on the development of malaria guidelines and recommendations. Endemic countries may also benefit from similar technical advisory committees to translate such the WHO recommendations into national strategic plans, including the consideration of relevant regulatory issues specific to malaria elimination, particularly for drugs and other tools that block transmission, but may have no particular benefit to the individual.

Implementation of elimination plans - Once the tools and strategies are developed and policy has incorporated them, the actual implementation of the specific elimination plan poses new and important challenges.

The first and most important issue to consider is that any elimination plan can only be implemented in the context of robust and responsive health systems. This means that, before embarking on a serious attempt to eliminate malaria, the needs of the national malaria program and the health system in general should be properly assessed following a check list that confirms that they are indeed ready.

Many researchers are currently working on such an evaluation framework. As it stands, this framework certainly will require the inclusion of a strong surveillance system that provides reliable and timely data to inform planning and action in real life and is capable of capturing hard to reach and mobile populations. Risk maps are also crucial for the elimination of malaria, both to assess the feasibility of the elimination goal itself in a given region and to provide data for stratification and intervention planning.

The other large component of an elimination plan is program management and logistics. No campaign can succeed unless the system is able to reach the targeted population, ensure stocks of commodities, adapt the activities to the surveillance data and manage the supply chain. In assessing such needs, countries should consider that staff needs may significantly increase within an elimination strategy, including personnel for the surveillance system and data analysis, field workers and, potentially, community-based volunteers. Overall, this component may affect the required budget and needs to be carefully considered when planning for malaria elimination.

All of these components necessarily lead to training and capacity-building in countries that are reorienting their programs towards elimination in the short or medium terms. Strengthening malaria programs from the human resources perspective is actually a recurrent request in country grants. Most signalled needs are training for entomologists, which is a field almost completely forgotten in recent years, but they also include training of personnel at all levels within the national health system.

In fact, what countries need most are experts with an integral and multidisciplinary knowledge of the disease, capable of making decisions and adapting the strategies to ever shifting epidemiological contexts. It has mockingly been observed that "the only thing the malaria eradication campaign really managed to eliminate was malariologists" (Alonso et al. 2011). Perhaps it is time to recall the old idea of schools of malariology distributed throughout the world and covering regions with similar epidemiological, political and economic challenges, to train those that ultimately will be responsible for designing and implementing malaria elimination strategies in their specific countries.

Students attending such schools of malariology would be trained in basic biology and parasitology, epidemiology and statistics, demography, pharmacology and drug strategies, diagnostics, entomology, social sciences and education, program planning and management, economy and financing, advocacy and communication, among other subjects and should clearly understand the specific challenges posed by the elimination approach.

The various meanings of sustainability - When talking about elimination, sustainability is perhaps the most cited and important concept. It refers to a number of issues in which thinking in the long term is crucial and above all, includes sustaining elimination itself in a malaria liberated territory (Chiyaka et al. 2013). Until global eradication is finally attained, the risk of reintroducing malaria in regions bordering areas where transmission still takes place is one of the most critical issues to be addressed.

Maps can play an important role in assessing such a risk and designing strategies to overcome it. Regional collaborative elimination approaches are also crucial in this aspect, as elimination is easier to be achieved if all countries in a defined region advance together towards this goal. Transborder collaborations should define interventions tailored to the specificities of the population flows (both legal and illegal) throughout countries, while strong surveillance systems ready to react in the event of a detected case need to be maintained as long as the risk of introduction exists.

In a preparatory stage, malaria elimination will require sustained funding and long-lasting political commitment as well as advocacy efforts to raise awareness of the specific challenges of addressing malaria from the point of view of elimination. Local health authorities need to be convinced of the feasibility and advantages of aiming for such a goal. In high transmission countries 
where elimination cannot be envisioned in the short term, they also should be reassured that committing to malaria elimination does not mean abandoning the control programs that they have already put in place, but rather working in parallel towards strengthening the system and creating capacities that will later allow malaria elimination.

In the meantime, clearing the malaria parasite from some defined subnational areas can play an important role in giving governments the confidence they need to embrace elimination. Economic analyses of the costs and benefits of elimination in the long term also can help in convincing authorities that such investments are worthwhile. The establishment of advocacy units specifically pushing for elimination, with key local or international figures nominated as malaria champions in the countries, also should be considered in the context of broader elimination and eradication strategies.

Also related to the long-term perspective is the recurrent problem in many endemic countries of the constant rotation of health authorities at the directorate level, which increases the difficulty of implementing long-lasting approaches such as those required by elimination. Some supra-governmental technical structures in the form of technical advisory committees may help to overcome this stability challenge ensuring continuity and taking the best advantage of the advice of malaria experts for the development of national elimination strategic plans.

At an international level, support should come from the WHO and the Roll Back Malaria Partnership (RBM). In addition to tracking global progress in the fight against malaria, mandates for the WHO Global Malaria Program include setting evidence-based policies and guidelines, developing approaches for the strengthening of malaria country programs and identifying new opportunities for action. The Global Technical Strategy for Malaria (2016-2025) that the WHO is currently preparing gives special emphasis to malaria elimination and the organisation shall play a crucial role in providing the best technical guidance to countries as per how to approach elimination.

On the other hand, using the forthcoming revised Global Malaria Action Plan, RBM should take the lead in the advocacy and communications issues related to elimination, aligning key international funding bodies, academia and product development partnerships, among other stakeholders, towards the final goal of malaria eradication as well as in fostering alliances that address elimination from a regional point of view. Regional networks for malaria elimination can in fact create synergies and reduce the overall risk of parasite reintroduction from neighbouring countries that are not advancing towards elimination at exactly the same pace. Technical support can also be provided in an easier manner to entire regions, while regional approaches also can benefit from agile teams that contribute to keeping malaria high in the national political agendas, even when cases are so low that they may no longer be considered a public health priority.

The steps forward - The potential benefits of eradicating malaria and the threatening scenario of resurgences and increases in the malaria burden, foreseen both by historical experience and modellers, urge the malaria community to reorient programs in areas where elimination is currently considered feasible and to set the stage for future elimination approaches in regions with too high transmission, too weak health systems or other currently unsurmountable epidemiological challenges.

In this process, wisdom is mandatory. We have to take advantage of natural bottle necks that make mosquitoes and parasites most vulnerable. We need to convince governments, policy makers and funding bodies about the relevance of the elimination approach to malaria and look for champions that put it high on the political, research and health agendas.

From a knowledge point of view, we need to generate evidence to inform field-rooted elimination strategies through the strengthening of the research component of elimination campaigns and the recovery of the so-called grey literature, as there are many informative experiences that have not been systematised in the form of scientific publications but that can still provide relevant insights and lessons.

Finally, learning from the past and other diseases elimination campaigns, we know today that we cannot address elimination in the hardest places until the end. Islands can move forward more or less on their own, but the true contribution to global malaria eradication is tackling the large areas where most of the malaria burden in the world is concentrated from an early stage, which very much coincides with countries included in the Malaria Situation Room: Burkina Faso, Cameroon, Côte d'Ivoire, the Democratic Republic of the Congo, Ghana, Mozambique, Niger, Nigeria, Uganda and Tanzania (WHO 2013). This applies at a global level, but it also has implications for national and regional planning. According to the same rationale, it will also be crucial to address hard to reach populations such as migrants, transborder populations, illegal workers and other mobile groups from the very beginning.

In any case, a first step in this effort will be to convince all involved parties that malaria control and elimination are not mutually exclusive. Both are equally necessary, as is also dealing simultaneously and with the same dedication and intensity, with $P$. falciparum and $P$. vivax. Despite the additional problems posed by dormant stages of $P$. vivax and relapses, it is also true that most countries that have achieved elimination were endemic predominantly with this species and that better drugs for radical cure of the dormant hypnozoites are currently being developed.

In light of the major gains achieved against malaria during the last decade and the threats associated to the relaxation of efforts, we cannot afford to throw away the unique opportunity we face today to advance towards elimination and ultimately eradication. The prize envisioned is impossible to underestimate: millions of lives saved, resources liberated and humanity free from one of the diseases that has caused the most suffering.

Panel 1 - Regional malaria elimination initiatives - According to the WHO classification, in December 2013, 12 countries were in the pre-elimination stage, seven in the elimination stage and seven in the prevention of reintroduction stage, with four countries having 
been certified malaria free since 2007. This is a reflection of the extraordinary advances in the fight against malaria during the last decade and the establishment of different regional malaria elimination networks.

Currently, these alliances include:

Initiative to Eliminate Malaria in Mesoamerica and the Hispaniola Island - In 2013, 10 countries in Central America and the Caribbean committed to eliminate malaria by 2020: Mexico, Belize, Guatemala, Honduras, El Salvador, Nicaragua, Costa Rica, Panama, Haiti and the Dominican Republic. Hispaniola is currently the only island of the Caribbean with malaria transmission, while Central America has made gigantic steps towards elimination and has the additional advantage of a population of parasites still sensitive to CQ.

Asia Pacific Malaria Elimination Network (APMEN) - Established in 2009, APMEN reunites 15 Asia Pacific Countries: Bhutan, Cambodia, China, Democratic People's Republic of Korea, Indonesia, Lao People's Democratic Republic, Malaysia, Nepal, Philippines, Republic of Korea, Solomon Islands, Sri Lanka, Thailand, Vanuatu and Vietnam. The main goals of APMEN are to eliminate malaria from the entire region in the long term and to achieve elimination by 2015 in half of the country partners. The area is particularly critical, as it has experienced the emergence of parasites resistant to artemisinin.

Elimination 8 - In 2009, eight countries in southern Africa launched this regional initiative with the goal of strengthening transborder collaborations, eliminating malaria by 2015 in Botswana, Namibia, South Africa and Swaziland and advancing towards elimination in the longer term in Angola, Mozambique, Zambia and Zimbabwe.

Economic Community of West African States Malaria Elimination Campaign - Launched in 2009, the initiative reunites 15 countries members: Benin, Burkina Faso, Cabo Verde, Côte d'Ivoire, Gambia, Ghana, Guiney, Guiney Bissau, Liberia, Mali, Niger, Nigeria, Senegal, Sierra Leone and Togo. The campaign aims at advancing towards malaria elimination mainly through an integrated vector control approach.

The Arabian Peninsula Free of Malaria Initiative - Under the umbrella of the Health Minister's Gulf Cooperation Council (GCC) (comprising Bahrain, Kuwait, Oman, Qatar, Saudi Arabia and the United Arab Emirates), this initiative was approved in 2007 with two key objectives: to interrupt transmission from endemic areas in Saudi Arabia and Yemen (which is not a member of GCC, but is located in the Arabian Peninsula) and to prevent the reestablishment of malaria transmission in areas that were already malaria-free through a network of epidemiological and entomological surveillance. The initiative addresses a region with high risk of reintroduction of malaria due to pilgrimage and transborder population movement.

The move from malaria control to elimination in the WHO European Region (Tashkent Declaration) - Nine countries in the European Region of the WHO committed in 2005 at scaling up the response to malaria with the ultimate objective of completely interrupting transmis- sion. The declaration was signed by Ministers of Health of Armenia, Azerbaijan, Georgia, Kazakhstan, Kyrgyzstan, Tajikistan, Turkey, Turkmenistan and Uzbekistan.

Sub Regional Malaria Elimination Programme Launched in 1997, this was the first regional initiative for the elimination of malaria and included the five northern African countries: Morocco, Algeria, Libya, Tunisia and Egypt. Among these countries, Morocco was certified malaria free by the WHO in 2010.

\section{REFERENCES}

Alonso PL, Brown G, Arévalo-Herrera M, Binka F, Chitnis C, Collins F, Doumbo OK, Greenwood B, Hall BF, Levine MM, Mendis K, Newman RD, Plowe CV, Rodríguez MH, Sinden R, Slutsker L, Tanner M 2011. A research agenda to underpin malaria eradication. PLoS Med 8: e1000406.

Bill \& Melinda Gates Foundation 2007. Malaria Forum Keynote Address. Available from: gatesfoundation.org/speeches-commentary/Pages/melinda-french-gates-2007-malaria-forum.aspx.

Breman JG, de Quadros CA, Dowdle WR, Foege WH, Henderson DA, John TJ, Levine MM 2011. The role of research in viral disease eradication and elimination programs: lessons for malaria eradication. PLoS Med 8: e1000405.

Chiyaka C, Tatem AJ, Cohen JM, Gething PW, Johnston G, Gosling R, Laxminarayan R, Hay SI, Smith DL 2013. The stability of malaria elimination. Science 339: 909-910.

malERA - Consultative Group on Basic Science and Enabling Technologies 2011. A research agenda for malaria eradication: basic science and enabling technologies. PLoS Med 8: e1000399.

Mendis K, Rietveld A, Warsame M, Bosman A, Greenwood B, Wernsdorfer WH 2009. From malaria control to eradication: the WHO perspective. Trop Med Int Health 14: 802-809.

MESA - Malaria Eradication Scientific Alliance 2011. ISGlobal: Barcelona. Available from: isglobal.org/en/web/guest/project// asset_publisher/qf6QOKuKkIC3/content/malaria-eradicationscientific-alliance-mesa-.

Moonen B, Cohen JM, Tatem AJ, Cohen J, Hay SI, Sabot O, Smith DL 2010. A framework for assessing the feasibility of malaria elimination. Malar J 9: 322 .

Nájera J, González-Silva M, Alonso PL 2011. Some lessons for the future from the Global Malaria Eradication Programme (19551969). PLoS Med 8: e1000412.

Newman RD 2012. Relegating malaria resurgences to history. Malar J 11: 123.

Policy Cures 2013. Estimating costs and measuring investments in malaria R\&D for eradication. Available from: policycures.org/ downloads/Estimating\%20Costs.pdf.

RBM Partnership - Roll Back Malaria Partnership 2011. Eliminating malaria: learning from the past, looking ahead. Available from: rbm.who.int/ProgressImpactSeries/docs/report9-en.pdf.

RTS,S Clinical Trials Partnership 2012. A Phase 3 trial of RTS,S/AS01 malaria vaccine in African infants. N Engl J Med 367: 2284-2295.

WHO - World Health Organization 2013. World malaria report. Available from: who.int/malaria/publications/world malaria report_2013/en/.

WHO - World Health Organization 2014. Planning meeting for operational research on malaria elimination. Meeting report. Available from: who.int/malaria/publications/atoz/operational-researchmalaria-elimination/en/. 